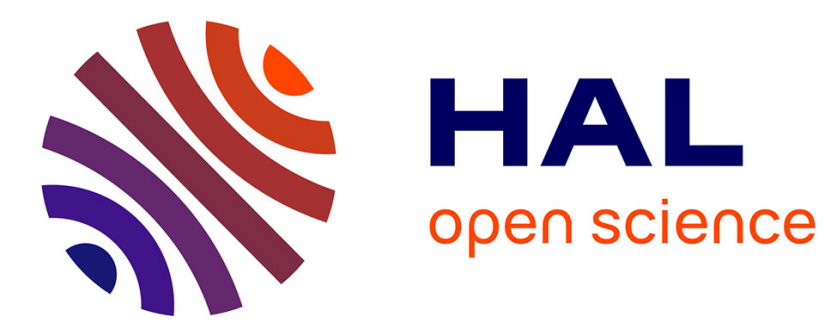

\title{
Theory and Calibration for Axial Cameras
}

Srikumar Ramalingam, Peter Sturm, Suresh K. Lodha

\section{To cite this version:}

Srikumar Ramalingam, Peter Sturm, Suresh K. Lodha. Theory and Calibration for Axial Cameras. Asian Conference on Computer Vision, Jan 2006, Hyderabad, India. pp.704-713. inria-00387131

\section{HAL Id: inria-00387131 https://hal.inria.fr/inria-00387131}

Submitted on 24 May 2009

HAL is a multi-disciplinary open access archive for the deposit and dissemination of scientific research documents, whether they are published or not. The documents may come from teaching and research institutions in France or abroad, or from public or private research centers.
L'archive ouverte pluridisciplinaire HAL, est destinée au dépôt et à la diffusion de documents scientifiques de niveau recherche, publiés ou non, émanant des établissements d'enseignement et de recherche français ou étrangers, des laboratoires publics ou privés. 


\title{
Theory and Calibration for Axial Cameras
}

\author{
Srikumar Ramalingam ${ }^{1 \& 2}$, Peter Sturm ${ }^{1}$, and Suresh K. Lodha ${ }^{2}$ \\ 1 INRIA Rhône-Alpes, Montbonnot St Martin, France \\ \{Srikumar.Ramalingam, Peter.Sturm\}@inrialpes.fr \\ 2 University of California, Santa Cruz, USA \\ \{lodha\}@soe.ucsc.edu
}

\begin{abstract}
Although most works in computer vision use perspective or other central cameras, the interest in non-central camera models has increased lately, especially with respect to omnidirectional vision. Calibration and structure-from-motion algorithms exist for both, central and non-central cameras. An intermediate class of cameras, although encountered rather frequently, has received less attention. So-called axial cameras are non-central but their projection rays are constrained by the existence of a line that cuts all of them. This is the case for stereo systems, many non-central catadioptric cameras and pushbroom cameras for example. In this paper, we study the geometry of axial cameras and propose a calibration approach for them. We also describe the various axial catadioptric configurations which are more common and less restrictive than central catadioptric ones. Finally we used simulations and real experiments to prove the validity of our theory.
\end{abstract}

\section{Introduction}

Many camera models have been considered in computer vision and related fields and even more taylor-made calibration methods have been developed. Most of those are designed for central cameras, but approaches and studies for noncentral or general ones also exist $[5-9,16,12,13,3]$. An intermediate class of cameras, lying between central and fully non-central ones, is that of so-called axial cameras: their projection rays are constrained by the existence of a line that cuts all of them, the camera axis, but they may not go through a single optical center.

The axial model is a rather useful one (cf. figure 1(a) and (b)). Many misaligned catadioptric configurations fall under this model. Such configurations, which are slightly non-central, are usually classified as a non-central camera and calibrated using an iterative nonlinear algorithm $[10,2,14]$. For example, whenever the mirror is a surface of revolution and the central camera looking at the mirror lies anywhere on the revolution axis, the system is of axial type. Furthermore, two-camera stereo systems or systems consisting of three or more aligned cameras, are axial. Pushbroom cameras [15] are another example, although they are of a more restricted class (there exist two camera axes [4]).

In this paper, we propose a generic calibration approach for axial cameras, the first to our knowledge. It uses images of planar calibration grids, put in unknown positions. We show the existence of multi-view tensors that can be 


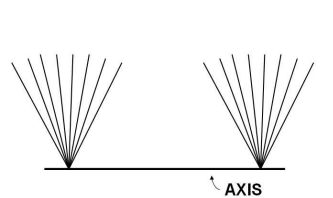

(a)

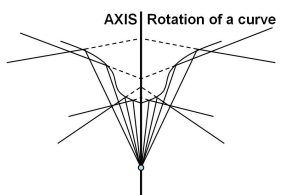

(b)

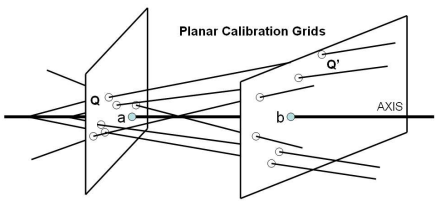

(c)

Fig. 1. Examples of axial imaging models (a) stereo camera (b) a mirror formed by rotating a planar curve about an axis containing the optical center of the perspective camera.(c) Calibration of axial cameras using calibration grids: The projection rays, camera axis and two grids are shown. The axis intersects at $\mathbf{a}$ and $\mathbf{b}$ on the first and the second calibration grids respectively.

estimated linearly and from which the pose of the calibration grids as well as the position of the camera axis, can be recovered. The actual calibration is then performed by computing projection rays for all individual pixels of a camera, constrained to cut the camera axis.

The paper is organized as follows. The problem is formalized in section 2 . In section 3, we show what can be done with two images of calibration grids. Complete calibration using three images, is described in section 4 , followed by a bundle adjustment algorithm in section 5 . Various types of axial catadioptric cameras are listed in section 6. Experimental results and conclusions are given in sections 7 and 8 .

\section{Problem Formulation}

In the following, we will call camera axis the line cutting all projection rays. It will be represented by a 6 -vector $\mathbf{L}$ and the associated $4 \times 4$ skew-symmetric Plücker matrix $[\mathbf{L}]_{\times}$:

$$
[\mathbf{L}]_{\times}=\left(\begin{array}{cccc}
0 & -L_{4} & L_{6} & -L_{2} \\
L_{4} & 0 & -L_{5} & -L_{3} \\
-L_{6} & L_{5} & 0 & -L_{1} \\
L_{2} & L_{3} & L_{1} & 0
\end{array}\right)
$$

The product $[\mathbf{L}]_{\times} \mathbf{Q}$ gives the plane spanned by the line $\mathbf{L}$ and the point $\mathbf{Q}$. Consider further the two 3 -vectors:

$$
\mathbf{A}=\left(\begin{array}{l}
L_{5} \\
L_{6} \\
L_{4}
\end{array}\right), \quad \mathbf{B}=\left(\begin{array}{l}
L_{2} \\
L_{3} \\
L_{1}
\end{array}\right)
$$


for which the Plücker constraint holds: $\mathbf{B}^{\top} \mathbf{A}=0$. $\mathbf{A}$ represents the point at infinity of the line. The Plücker matrix can be written as:

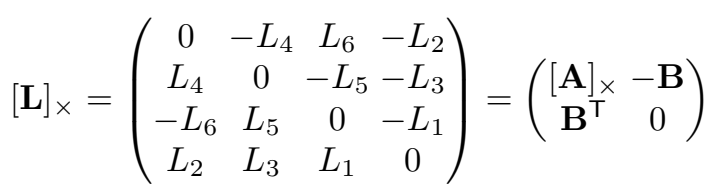

The calibration problem considered in this paper is to compute projection rays for all pixels of a camera, from images of planar calibration grids in unknown positions. We assume that dense point correspondences are given, i.e. for (many) pixels, we are able to determine the points on the calibration grids that are seen in that pixel. Computed projection rays will be constrained to cut the camera axis. The coordinate system in which calibration will be expressed, is that of the first calibration grid. Calibration thus consists in computing the position of the camera axis and of the projection rays, in that coordinate system. The proposed approach proceeds by first estimating the camera axis and the pose of all grids but the first one.

\section{What can be Done with Two Views of Calibration Grids?}

Consider some pixel and let $\mathbf{Q}$ and $\mathbf{Q}^{\prime}$ be the corresponding points on the two calibration grids, given as 3D points in the grids' local coordinate systems. Since we consider planar grids, we impose $Q_{3}=Q_{3}^{\prime}=0$.

We have the following constraint on the pose of the second grid $\left(R^{\prime}, \mathbf{t}^{\prime}\right)$ as well as the unknown camera axis $\mathbf{L}$ : the line spanned by $\mathbf{Q}$ and $\mathbf{Q}^{\prime}$ cuts $\mathbf{L}$, hence is coplanar with it. Hence, for the correct pose and camera axis, we must have:

$$
\mathbf{Q}^{\top}[\mathbf{L}]_{\times}\left(\begin{array}{cc}
\mathbf{R}^{\prime} & \mathbf{t}^{\prime} \\
\mathbf{0}^{\top} & 1
\end{array}\right) \mathbf{Q}^{\prime}=0
$$

Hence:

$$
\left(\begin{array}{l}
Q_{1} \\
Q_{2} \\
Q_{4}
\end{array}\right)^{\top}\left(\begin{array}{cccc}
0 & -L_{4} & L_{6} & -L_{2} \\
L_{4} & 0 & -L_{5} & -L_{3} \\
L_{2} & L_{3} & L_{1} & 0
\end{array}\right)\left(\begin{array}{cc}
\overline{\mathrm{R}^{\prime}} & \mathbf{t}^{\prime} \\
\mathbf{0}^{\top} & 1
\end{array}\right)\left(\begin{array}{l}
Q_{1}^{\prime} \\
Q_{2}^{\prime} \\
Q_{4}^{\prime}
\end{array}\right)=0
$$

where $\overline{\mathrm{R}^{\prime}}$ refers to the $3 \times 2$ submatrix of $\mathrm{R}^{\prime}$ containing only the first and the second rows. We thus have the following $3 \times 3$ tensor that can be estimated linearly from point correspondences:

$$
\mathrm{F} \sim\left(\begin{array}{cccc}
0 & -L_{4} & L_{6} & -L_{2} \\
L_{4} & 0 & -L_{5} & -L_{3} \\
L_{2} & L_{3} & L_{1} & 0
\end{array}\right)\left(\begin{array}{cc}
\overline{\mathrm{R}^{\prime}} & \mathbf{t}^{\prime} \\
\mathbf{0}^{\top} & 1
\end{array}\right)
$$

It has only 7 degrees of freedom ( 9 - 1 for scale, -1 for rank-deficiency) so the 10 unknowns ( 4 for the camera axis, 3 for $\mathrm{R}^{\prime}$ and 3 for $\mathbf{t}^{\prime}$ ) can not be recovered from it. 
We now look at what can actually be recovered from $F$. Let us first notice that its left null-vector is $\left(L_{3},-L_{2}, L_{4}\right)^{\top}$ (it truly is the null-vector, as can be easily verified when taking into account the Plücker constraint). We thus can recover 2 of the 4 parameters of the camera axis. That null-vector contains actually the coordinates of the camera axis' intersection with the first grid (in plane coordinates). Its $3 \mathrm{D}$ coordinates are given by $\left(L_{3},-L_{2}, 0, L_{4}\right)^{\top}$. Similarly, the right null-vector of $\mathrm{F}$ gives the plane coordinates of the axis' intersection with the second grid. Besides this $F$ also gives constraints on $R^{\prime}$ and $\mathbf{t}^{\prime}$. For example $\mathrm{R}^{\prime}$ can be extracted up to 2 to 4 solutions. We will later observe that once we locally shift the intersection points, between the camera axis and calibration grids, to the origins of the respective grids the vector $\mathbf{t}^{\prime}$ will lie on the camera axis. Inspite of all these additional constraints, arising from axial geometry, two views of calibration grids are not sufficient to uniquely extract $R^{\prime}$ and $\mathbf{t}^{\prime}$. Thus we use three calibration grids as described below.

\section{Full Calibration using Three Views of Calibration Grids}

Let $\mathbf{Q}, \mathbf{Q}^{\prime}, \mathbf{Q}^{\prime \prime}$ refer to the grid points corresponding to a single pixel in the three grids. The poses of the grids are $(I, 0),\left(R^{\prime}, t^{\prime}\right)$ and $\left(R^{\prime \prime}, t^{\prime \prime}\right)$ respectively. Since the three points $\mathbf{Q}, \mathbf{Q}^{\prime}$ and $\mathbf{Q}^{\prime \prime}$ are collinear we use this constraint to extract the poses of the calibration grids [12]. Every $3 \times 3$ submatrix of the following $4 \times 3$ matrix has zero subdeterminant.

$$
\left(\begin{array}{lll}
\mathbf{Q} & \left(\begin{array}{ll}
\mathrm{R}^{\prime} & \mathbf{t}^{\prime} \\
\mathbf{0}^{\top} & 1
\end{array}\right) \mathbf{Q}^{\prime} \quad\left(\begin{array}{cc}
\mathrm{R}^{\prime \prime} & \mathbf{t}^{\prime \prime} \\
\mathbf{0}^{\mathrm{T}} & 1
\end{array}\right) \mathbf{Q}^{\prime \prime}
\end{array}\right)
$$

The submatrices constructed by removing the first and the second rows lead to the constraints $\sum C_{i} T 1_{i}=0$ and $\sum C_{i} T 2_{i}=0$ respectively (as described in Table 1). These are nothing but homogeneous linear systems of the form $A X=0$. The unknown vector $X$ is formed from the 14 variables $\left(C_{i}\right)$. Each of these variables are coupled coefficients of the poses of the grids. The matrix $A$ is constructed by stacking the trilinear tensors $T 1$ and $T 2$, which can be computed from the coordinates of $\mathbf{Q}, \mathbf{Q}^{\prime}$ and $\mathbf{Q}^{\prime \prime}$. In future when we refer to the rank of a linear system $A X=0$, we refer to the rank of the matrix $A$. The rank has to be one less than the number of variables to estimate them uniquely upto a scale. For example, each of the above linear systems must have a rank of 13 to estimate the coefficients $\left(C_{i}\right)$ uniquely. These systems were used to calibrate completely non-central cameras [10]. However in the case of axial cameras, these systems were found to have a rank of 12 . This implies that the solution can not be obtained uniquely. In order to resolve this ambiguity we will need more constraints.

\subsection{Intersection of axis and calibration grids}

Using the technique described earlier we compute the intersection of the camera axis with the three grids at $\mathbf{a}, \mathbf{b}$ and $\mathbf{c}$ respectively. We translate the local 


\begin{tabular}{|r|r|r|r||r|r|r|r|}
\hline $\mathrm{i}$ & Motion $\left(C_{i}\right)$ & $T 1_{i}$ & $T 2_{i}$ & $\mathrm{i}$ & Motion $\left(C_{i}\right)$ & $T 1_{i}$ & $T 2_{i}$ \\
\hline \hline 1 & $R_{31}^{\prime}$ & $Q_{2} Q_{1}^{\prime} Q_{4}^{\prime \prime}$ & $Q_{1} Q_{1}^{\prime} Q_{4}^{\prime \prime}$ & 13 & $R_{22}^{\prime} R_{32}^{\prime \prime}-R_{32}^{\prime} R_{22}^{\prime \prime}$ & $Q_{4} Q_{2}^{\prime} Q_{2}^{\prime \prime}$ & 0 \\
\hline 2 & $R_{32}^{\prime}$ & $Q_{2} Q_{2}^{\prime} Q_{4}^{\prime \prime}$ & $Q_{1} Q_{2}^{\prime} Q_{4}^{\prime \prime}$ & 14 & $R_{11}^{\prime} t_{3}^{\prime \prime}-R_{33}^{\prime} t_{1}^{\prime \prime}$ & 0 & $Q_{4} Q_{1}^{\prime} Q_{4}^{\prime \prime}$ \\
\hline 3 & $R_{31}^{\prime \prime}$ & $-Q_{2} Q_{4}^{\prime} Q_{1}^{\prime \prime}$ & $-Q_{1} Q_{4}^{\prime} Q_{1}^{\prime \prime}$ & 15 & $R_{12}^{\prime} t_{3}^{\prime \prime}-R_{32}^{\prime} t_{1}^{\prime \prime}$ & 0 & $Q_{4} Q_{2}^{\prime} Q_{4}^{\prime \prime}$ \\
\hline 4 & $R_{32}^{\prime \prime}$ & $-Q_{2} Q_{4}^{\prime} Q_{2}^{\prime \prime}$ & $-Q_{1} Q_{4}^{\prime} Q_{2}^{\prime \prime}$ & 16 & $R_{21}^{\prime} t_{3}^{\prime \prime}-R_{31}^{\prime} t_{2}^{\prime \prime}$ & $Q_{4} Q_{1}^{\prime} Q_{4}^{\prime \prime}$ & 0 \\
\hline 5 & $t_{3}^{\prime}-t_{3}^{\prime \prime}$ & $Q_{2} Q_{4}^{\prime} Q_{4}^{\prime \prime}$ & $Q_{1} Q_{4}^{\prime} Q_{4}^{\prime \prime}$ & 17 & $R_{21}^{\prime} t_{3}^{\prime \prime}-R_{32}^{\prime} t_{2}^{\prime \prime}$ & $Q_{4} Q_{2}^{\prime} Q_{4}^{\prime \prime}$ & 0 \\
\hline 6 & $R_{11}^{\prime} R_{31}^{\prime \prime}-R_{31}^{\prime} R_{11}^{\prime \prime}$ & 0 & $Q_{4} Q_{1}^{\prime} Q_{1}^{\prime \prime}$ & 18 & $R_{11}^{\prime \prime} t_{3}^{\prime}-R_{33}^{\prime \prime} t_{1}^{\prime}$ & 0 & $-Q_{4} Q_{4}^{\prime} Q_{1}^{\prime \prime}$ \\
\hline 7 & $R_{11}^{\prime} R_{32}^{\prime \prime}-R_{31}^{\prime} R_{12}^{\prime \prime}$ & 0 & $Q_{4} Q_{1}^{\prime} Q_{2}^{\prime \prime}$ & 19 & $R_{12}^{\prime \prime} t_{3}^{\prime}-R_{33}^{\prime \prime} t_{1}^{\prime}$ & 0 & $-Q_{4} Q_{4}^{\prime} Q_{2}^{\prime \prime}$ \\
\hline 8 & $R_{12}^{\prime} R_{31}^{\prime \prime}-R_{32}^{\prime} R_{11}^{\prime \prime}$ & 0 & $Q_{4} Q_{2}^{\prime} Q_{1}^{\prime \prime}$ & 20 & $R_{21}^{\prime \prime} t_{3}^{\prime}-R_{33}^{\prime \prime} t_{2}^{\prime}$ & $-Q_{4} Q_{4}^{\prime} Q_{1}^{\prime \prime}$ & 0 \\
\hline 9 & $R_{12}^{\prime} R_{32}^{\prime \prime}-R_{32}^{\prime} R_{12}^{\prime \prime}$ & 0 & $Q_{4} Q_{2}^{\prime} Q_{2}^{\prime \prime}$ & 21 & $R_{22}^{\prime \prime} t_{3}^{\prime}-R_{33}^{\prime \prime} t_{2}^{\prime}$ & $-Q_{4} Q_{4}^{\prime} Q_{2}^{\prime \prime}$ & 0 \\
\hline 10 & $R_{21}^{\prime} R_{31}^{\prime \prime}-R_{31}^{\prime} R_{21}^{\prime \prime}$ & $Q_{4} Q_{1}^{\prime} Q_{1}^{\prime \prime}$ & 0 & 22 & $t_{1}^{\prime} t_{3}^{\prime \prime}-t_{3}^{\prime} t_{1}^{\prime \prime}$ & 0 & $Q_{4} Q_{4}^{\prime} Q_{4}^{\prime \prime}$ \\
\hline 11 & $R_{21}^{\prime} R_{32}^{\prime \prime}-R_{31}^{\prime} R_{22}^{\prime \prime}$ & $Q_{4} Q_{1}^{\prime} Q_{2}^{\prime \prime}$ & 0 & 23 & $t_{2}^{\prime} t_{3}^{\prime \prime}-t_{3}^{\prime} t_{2}^{\prime \prime}$ & $Q_{4} Q_{4}^{\prime} Q_{4}^{\prime \prime}$ & 0 \\
\hline 12 & $R_{22}^{\prime} R_{31}^{\prime \prime}-R_{32}^{\prime} R_{21}^{\prime \prime}$ & $Q_{4} Q_{2}^{\prime} Q_{1}^{\prime \prime}$ & 0 & 0 & & & \\
\hline
\end{tabular}

Table 1. Trifocal tensor in the generic calibration of completely non-central cameras.

grid coordinates such that these intersection points become their respective origins. Without loss of generality we continue to use the same notations after the transformations.

$$
\mathbf{Q} \longleftarrow \mathbf{Q}-\mathbf{a}, \mathbf{Q}^{\prime} \longleftarrow \mathbf{Q}^{\prime}-\mathbf{b}, \mathbf{Q}^{\prime \prime} \longleftarrow \mathbf{Q}^{\prime \prime}-\mathbf{c},
$$

We can obtain a collinearity constraint by putting these origins in the same coordinate system. Every $3 \times 3$ subdeterminant of the following $4 \times 3$ matrix vanishes.

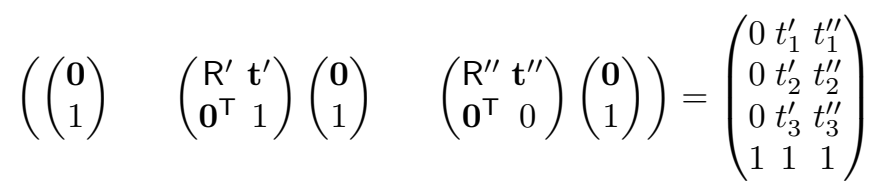

The camera axis passes through $O, \mathbf{t}^{\prime}$ and $\mathbf{t}^{\prime \prime}$. This enables us to express $\mathbf{t}^{\prime \prime}$ as a multiple of $\mathbf{t}^{\prime}$ using some scalar $\Delta: \mathbf{t}^{\prime \prime}=\Delta \mathbf{t}^{\prime}$. As a result, the variables $C_{22}$ and $C_{23}$ from Table 1 disappear.

$$
\begin{aligned}
& C_{22}=t_{1}^{\prime} t_{3}^{\prime \prime}-t_{3}^{\prime} t_{1}^{\prime \prime}=t_{1}^{\prime} \Delta t_{3}^{\prime}-t_{3}^{\prime} \Delta t_{1}^{\prime}=0 \\
& C_{23}=t_{2}^{\prime} t_{3}^{\prime \prime}-t_{3}^{\prime} t_{2}^{\prime \prime}=t_{2}^{\prime} \Delta t_{3}^{\prime}-t_{3}^{\prime} \Delta t_{2}^{\prime}=0
\end{aligned}
$$

On disappearing, $C_{22}$ and $C_{23}$ reduce the size of the linear systems $\sum C_{i} T 1_{i}=0$ and $\sum C_{i} T 2_{i}=0$ each by one. Inspite of this reduction there still exists a rank deficiency of 2 in both these systems. The rank of each of these systems is 11 with 13 nonzero coefficients to be estimated. In the next section we provide the details of the usage of a coplanarity constraint, which exists in axial cameras, to remove the degeneracy problems.

\subsection{Coplanarity constraints in axial cameras}

The camera axis cuts all the projection rays. As observed earlier both $\mathbf{O}$ and $\mathbf{t}^{\prime}$ lie on the camera axis. Along with these two points, we consider two grid points 
$\mathbf{Q}^{\prime}$ and $\mathbf{Q}^{\prime \prime}$ lying on a single projection ray. Since these four points are coplanar, the determinant of the following $4 \times 4$ matrix disappears.

$$
\left.\left(\begin{array}{l}
0 \\
0 \\
0 \\
1
\end{array}\right) \quad\left(\begin{array}{c}
t_{1}^{\prime} \\
t_{2}^{\prime} \\
t_{3}^{\prime} \\
1
\end{array}\right) \quad\left(\begin{array}{cc}
\mathrm{R}^{\prime} & \mathbf{t}^{\prime} \\
\mathbf{0}^{\top} & 1
\end{array}\right) \mathbf{Q}^{\prime} \quad\left(\begin{array}{cc}
\mathrm{R}^{\prime \prime} & \boldsymbol{\Delta} t^{\prime} \\
\mathbf{0}^{\top} & 1
\end{array}\right) \mathbf{Q}^{\prime \prime}\right)
$$

The corresponding constraint is a linear system $\sum \alpha_{i j} Q_{i}^{\prime} Q_{j}^{\prime \prime}=0$ (see table 2). Note that $Q_{4}^{\prime}$ and $Q_{4}^{\prime \prime}$ are not present because of the three zeros in the first column. We can solve this linear system to computer the solutions for $\alpha_{i j}$. We expand the above linear system and do some algebraic manipulation.

$$
\begin{gathered}
\alpha_{11} Q_{1}^{\prime} Q_{1}^{\prime \prime}+\alpha_{12} Q_{1}^{\prime} Q_{2}^{\prime \prime}+\alpha_{21} Q_{2}^{\prime} Q_{1}^{\prime \prime}+\alpha_{22} Q_{2}^{\prime} Q_{2}^{\prime \prime}=0 \\
Q_{4}\left(\alpha_{11} Q_{1}^{\prime} Q_{1}^{\prime \prime}+\alpha_{12} Q_{1}^{\prime} Q_{2}^{\prime \prime}+\alpha_{21} Q_{2}^{\prime} Q_{1}^{\prime \prime}+\alpha_{22} Q_{2}^{\prime} Q_{2}^{\prime \prime}\right)=0 \\
Q_{4} Q_{2}^{\prime} Q_{2}^{\prime \prime}=-\frac{\alpha_{11}}{\alpha_{22}} Q_{4} Q_{1}^{\prime} Q_{1}^{\prime \prime}-\frac{\alpha_{12}}{\alpha_{22}} Q_{4} Q_{1}^{\prime} Q_{2}^{\prime \prime}-\frac{\alpha_{21}}{\alpha_{22}} Q_{4} Q_{2}^{\prime} Q_{1}^{\prime \prime}
\end{gathered}
$$

This will enable us to represent both $T 2_{9}$ and $T 1_{13}$, from the earlier systems, in terms of other variables in the tensors $T 1$ and $T 2$ respectively.

$$
\begin{gathered}
T 2_{9}=-\frac{\alpha_{11}}{\alpha_{22}} T 2_{6}-\frac{\alpha_{12}}{\alpha_{22}} T 2_{7}-\frac{\alpha_{21}}{\alpha_{22}} T 2_{8} \\
T 1_{13}=-\frac{\alpha_{11}}{\alpha_{22}} T 1_{10}-\frac{\alpha_{12}}{\alpha_{22}} T 1_{11}-\frac{\alpha_{21}}{\alpha_{22}} T 1_{12}
\end{gathered}
$$

Using the above relation we obtain two new constraints given by $\sum A_{i} A 1_{i}=0$

\begin{tabular}{|c|c|}
\hline \multicolumn{2}{|r|}{$\alpha_{i j}$} \\
\hline & $t_{1}^{\prime}\left(R_{2,1}^{\prime} R_{3,1}^{\prime \prime}-R_{2,1}^{\prime \prime} R_{3,1}^{\prime}\right)-t_{2}^{\prime}\left(R_{1,1}^{\prime} R_{3,}^{\prime \prime}\right.$ \\
\hline & $\frac{t_{1}^{\prime}\left(R_{2,1}^{\prime} R_{3,2}^{\prime \prime}-R_{2,2}^{\prime \prime} R_{3,1}^{\prime}\right)-t_{2}^{\prime}\left(R_{1,1}^{\prime} R_{3,2}^{\prime \prime}-R_{1,2}^{\prime \prime} R_{3,1}^{\prime}\right)+t_{3}^{\prime}\left(R_{1,1}^{\prime} R_{2,2}^{\prime \prime}-R_{1,2}^{\prime \prime} R_{2,1}^{\prime}\right)}{t_{1}^{\prime}\left(R_{2,}^{\prime} R_{3,}^{\prime \prime}-R_{2,1}^{\prime \prime} R_{3,2}^{\prime}\right)-t_{2}^{\prime}\left(R_{1,2}^{\prime} R_{3,1}^{\prime \prime}-R_{1,}^{\prime \prime} R_{3,}^{\prime}\right)+t_{3}^{\prime}\left(R_{1,}^{\prime} R_{2,1}^{\prime \prime}-R_{1,1}^{\prime \prime} R_{2}^{\prime}\right)}$ \\
\hline & 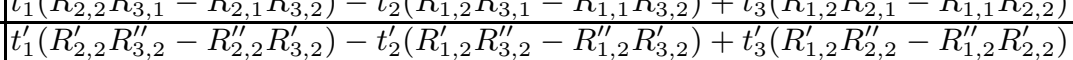 \\
\hline
\end{tabular}
and $\sum A_{i} A 2_{i}=0$. Note that each of these constraints are linear systems with 12 nonzero coefficients each. Both of them have a rank of 11 and thereby producing unique solutions for their coefficients $\left(A_{i}\right)$. The individual elements in the poses of the grids are extracted from these coupled coefficients using orthonormality constraints of the rotation matrix [12].

Table 2. Bifocal tensor from the coplanarity constraint on $\mathbf{O}, \mathbf{t}^{\prime}, \mathbf{Q}^{\prime}$ and $\mathbf{Q}^{\prime \prime}$.

\section{Bundle Adjustment Formulation}

We give the details of a bundle adjustment which refines the estimated camera axis and poses of the calibration grids. This is similar to our earlier method [10], except that we have an additional constraint coming from the camera axis. The 


\begin{tabular}{|r|r|r|r||r|r|r|r|}
\hline i & Motion $\left(A_{i}\right)$ & $A 1_{i}$ & $A 2_{i}$ & i & Motion $\left(A_{i}\right)$ & $A 1_{i}$ & $A 2_{i}$ \\
\hline \hline 1 & $R_{31}^{\prime}$ & $Q_{2} Q_{1}^{\prime} Q_{4}^{\prime \prime}$ & $Q_{1} Q_{1}^{\prime} Q_{4}^{\prime \prime}$ & 11 & $C_{12}-\frac{\alpha_{21}}{\alpha_{22}} C_{13}$ & $Q_{4} Q_{2}^{\prime} Q_{1}^{\prime \prime}$ & 0 \\
\hline 2 & $R_{32}^{\prime}$ & $Q_{2} Q_{2}^{\prime} Q_{4}^{\prime \prime}$ & $Q_{1} Q_{2}^{\prime} Q_{4}^{\prime \prime}$ & 12 & $\Delta\left(R_{11}^{\prime} t_{3}^{\prime}-R_{31}^{\prime} t_{1}^{\prime}\right)$ & 0 & $Q_{4} Q_{1}^{\prime} Q_{4}^{\prime \prime}$ \\
\hline 3 & $R_{31}^{\prime \prime}$ & $-Q_{2} Q_{4}^{\prime} Q_{1}^{\prime \prime}$ & $-Q_{1} Q_{4}^{\prime} Q_{1}^{\prime \prime}$ & 13 & $\Delta\left(R_{12}^{\prime} t_{3}^{\prime}-R_{32}^{\prime} t_{1}^{\prime}\right)$ & 0 & $Q_{4} Q_{2}^{\prime} Q_{4}^{\prime \prime}$ \\
\hline 4 & $R_{32}^{\prime \prime}$ & $-Q_{2} Q_{4}^{\prime} Q_{2}^{\prime \prime}$ & $-Q_{1} Q_{4}^{\prime} Q_{2}^{\prime \prime}$ & 14 & $\Delta\left(R_{21}^{\prime} t_{3}^{\prime}-R_{31}^{\prime} t_{2}^{\prime}\right)$ & $Q_{4} Q_{1}^{\prime} Q_{4}^{\prime \prime}$ & 0 \\
\hline 5 & $t_{3}^{\prime}-t_{3}^{\prime \prime}$ & $Q_{2} Q_{4}^{\prime} Q_{4}^{\prime \prime}$ & $Q_{1} Q_{4}^{\prime} Q_{4}^{\prime \prime}$ & 15 & $\Delta\left(R_{22}^{\prime} t_{3}^{\prime}-R_{32}^{\prime} t_{2}^{\prime}\right)$ & $Q_{4} Q_{2}^{\prime} Q_{4}^{\prime \prime}$ & 0 \\
\hline 6 & $C_{6}-\frac{\alpha_{11}}{\alpha_{22}} C_{9}$ & 0 & $Q_{4} Q_{1}^{\prime} Q_{1}^{\prime \prime}$ & 16 & $R_{11}^{\prime \prime} t_{3}^{\prime}-R_{33}^{\prime \prime} t_{1}^{\prime}$ & 0 & $-Q_{4} Q_{4}^{\prime} Q_{1}^{\prime \prime}$ \\
\hline 7 & $C_{7}-\frac{\alpha_{12} 2}{\alpha_{22} C_{9}}$ & 0 & $Q_{4} Q_{1}^{\prime} Q_{2}^{\prime \prime}$ & 17 & $R_{12}^{\prime \prime} t_{3}^{\prime}-R_{32}^{\prime \prime} t_{1}^{\prime}$ & 0 & $-Q_{4} Q_{4}^{\prime} Q_{2}^{\prime \prime}$ \\
\hline 8 & $C_{8}-\frac{\alpha_{21}}{\alpha_{22}} C_{9}$ & 0 & $Q_{4} Q_{2}^{\prime} Q_{1}^{\prime \prime}$ & 18 & $R_{22}^{\prime \prime} t_{3}^{\prime}-R_{33}^{\prime \prime} t_{2}^{\prime}$ & $-Q_{4} Q_{4}^{\prime} Q_{1}^{\prime \prime}$ & 0 \\
\hline 9 & $C_{10}-\frac{\alpha_{11}}{\alpha_{22}} C_{13}$ & $Q_{4} Q_{1}^{\prime} Q_{1}^{\prime \prime}$ & 0 & 19 & $R_{22}^{\prime \prime} t_{3}^{\prime}-R_{32}^{\prime \prime} t_{2}^{\prime}$ & $-Q_{4} Q_{4}^{\prime} Q_{2}^{\prime \prime}$ & 0 \\
\hline 10 & $C_{11}-\frac{\alpha_{12}}{\alpha_{22}} C_{13}$ & $Q_{4} Q_{1}^{\prime} Q_{2}^{\prime \prime}$ & 0 & & & & 0 \\
\hline
\end{tabular}

Table 3. Trifocal tensor for the generic calibration of axial cameras.

bundle adjustment is done by minimizing the distance between the grid points and the corresponding projection rays. The cost function is given below.

$$
\text { Cost }=\sum_{i=1}^{n} \sum_{j=1}\left(\mathbf{A}+\lambda_{i} \mathbf{D}+\mu_{j i} \mathbf{D}_{i}-\left[\mathrm{R}_{j} \mathbf{T}_{j}\right] \mathbf{Q}_{j i}\right)
$$

- $(\mathbf{A}, \mathbf{D})$ - represents the axis (point, direction)

- $\mathbf{D}_{i}$ - unit direction vector of the $i_{t h}$ projection ray

- $\lambda_{i}$ - parameter selecting the intersection of the $i_{t h}$ ray and the axis

- $\mathbf{Q}_{j i}$ - grid point on the $j_{t h}$ grid lying the $i_{t h}$ ray

- $\mu_{j i}$ - parameter selecting the point on the $i_{t h}$ ray closest to $Q_{j}$

- $\left(\mathrm{R}_{j}, \mathbf{T}_{j}\right)$ - pose of the calibration grid

\section{Axial Catadioptric Configurations}

Our formulation can classify a given camera into either axial or not. For example on applying our method on axial data we obtain unique solutions. On the other hand, a completely non-central camera will lead to an inconsistent (no solution), whereas a central camera will produce a rank deficient system (ambiguous solutions). Thus our technique produces unique solutions only for axial configurations. This can be used as a simple test in simulations to study the nature of complex catadioptric arrangements (as shown in Figure 2(a)). Since axial cameras are less restrictive than central cameras, they can be easily constructed using various combinations of mirrors and lenses. For example there are very few central configurations [1] (also see Table 4). Furthermore these configurations are difficult to build and maintain. For example, in a central catadioptric camera with hyperbolic mirror and perspective camera, the optical center has to be placed precisely on one of the mirror's focal points. On the other hand, the optical center can be anywhere on the mirror axis to have an axial geometry. 


\begin{tabular}{|c|c|c|c||c|c|c|}
\hline mirror & ctrl (pers) & axial (pers) & nctrl (pers) & ctral (ortho) & axial (ortho) & nctrl (ortho) \\
\hline \hline hyperbolic & $\mathrm{o}=\mathrm{f}$ & $\mathrm{o} \in \mathrm{MA}$ & $\mathrm{o} \notin \mathrm{MA}$ & - & OA $\| \mathrm{MA}$ & OA MA \\
\hline spherical & - & always & - & - & always & - \\
\hline parabolic & - & $\mathrm{o} \in \mathrm{MA}$ & $\mathrm{o} \notin \mathrm{MA}$ & $\mathrm{OA} \| \mathrm{MA}$ & - & OA MA \\
\hline elliptic & $\mathrm{o}=\mathrm{f}$ & $\mathrm{o} \in \mathrm{MA}$ & $\mathrm{o} \notin \mathrm{MA}$ & - & $\mathrm{OA} \| \mathrm{MA}$ & OA MA \\
\hline cone & - & $\mathrm{o} \in \mathrm{MA}$ & $\mathrm{o} \notin \mathrm{MA}$ & - & OA $\| \mathrm{MA}$ & OA $\nVdash \mathrm{MA}$ \\
\hline planar & always & - & planar & - & - & - \\
\hline mir-rot & - & always & - & - & always & - \\
\hline
\end{tabular}

Table 4. Catadioptric configurations. Notations: ctrl (pers) - central configuration with perspective camera, nctrl (ortho) - non-central configuration with orthographic camera, mir-rot - mirror obtained by rotating a planar curve about the optical axis, o - optical center of the perspective camera, $f$ - focus of the mirror, MA - major axis of mirror, OA - optical axis of the camera, = refers to same location, $\in$-lies on, $\|$-parallel, ł-not parallel.

\section{Experiments}

\subsection{Simulation}

We started with perfect axial configurations for three scenarios (as shown in Figures 2(a), (b) and (c)) and gradually change the configurations to make them non-central. We quantify this change from the perfect axial configuration as disparity. For example, in Figure 2(a), the disparity represents the distance between the optical center of the perspective camera and the orthographic camera axis passing through the center of the sphere. This optical center is initially at a distance of 3 units from the center of the sphere (which is of radius 1 unit). In Figure 2(b), the disparity represents the distance between the optical center of the perspective camera and the major axis of the hyperboloid. Initially the optical center is at a distance of 5 units from the tip of the hyperboloid, whose two radii are 5 and 10 units. In Figure 2(c), the disparity represents the distance between the optical center of the third camera and the line joining the first two cameras. The distance between two consecutive centers of the cameras is 40 units. We calibrate these systems in the presence of disparities. We compute the mean angular error between the original and the reconstructed projection rays in Figure 2(d). Note that the the mean angular error (given in radians) reaches zero only at the precise axial configuration.

\subsection{Stereo camera}

We captured three images of a calibration grid using two different cameras. The goal is to reconstruct the projection rays of both the cameras in the same generic framework using our axial calibration algorithm. Here the camera axis is the line joining the two optical centers (see Figure 3(a)). The image of the combined system is formed by concatenating the images from the two cameras. Figure 2(d) shows that our algorithm is very sensitive to noise. However using 


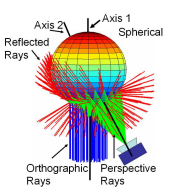

(a)

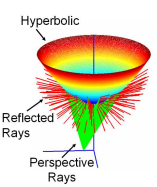

(b)

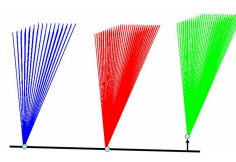

(c)

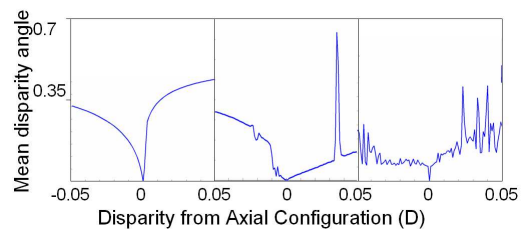

(d)

Fig. 2. Test for axial configuration. (a) Catadioptric (spherical mirror+pers.camera+ortho.camera): becomes non-central when the two optical centers and the sphere center are not collinear (as shown).(b) Catadioptric (Hyperbolic mirror+pers.camera): becomes non-central if the optical center is not on the axis of the hyperbolic mirror (as shown). (c) Tristereo when one of the cameras is axially misplaced (as shown). (d) shows the mean angular error between the original and reconstructed projection rays w.r.t disparity. The graphs shown in left, middle and right correspond to scenarios in (a), (b) and (c) respectively (see text for more details).

RANSAC, it is possible to obtain a good calibration. Once we compute the pose of the grids we can compute the rays corresponding to individual cameras in the stereo system. These rays can also be made to intersect separately and parameterized using a pinhole model. The RMS bundle adjustment error, based on the distance between the projection rays and grid points on the calibration grids, is of the order of $0.29 \%$ w.r.t overall size of the scene. The estimated camera parameters are close to the correct results. The reconstructed projection rays and grids are shown in Figure 3(a).

\subsection{Spherical catadioptric cameras}

We calibrated a real spherical catadioptric camera and extracted the camera axis. We start with an initial calibration using three grids using the above axial algorithm. This enables us to obtain an initial estimate for the axis and the projection rays. Using this partial calibration, we use pose estimation to incrementally compute the pose of newer grids. We followed our earlier method to obtain complete calibration [10]. The calibration grid captured by a spherical catadioptric camera is shown in Figure 3(b). We estimated the pose of several grids on a turntable sequence using the calibration. The grid positions and the axis are shown in Figure 3(c). For more details about results and other experimental issues please refer to [11].

\section{Conclusions}

We studied the theory and proposed a linear calibration algorithm for an intermediate class of cameras called axial cameras. Further line of investigation needs to be carried out to test the accuracy of this approach with respect to parametric and completely non-central approaches. 


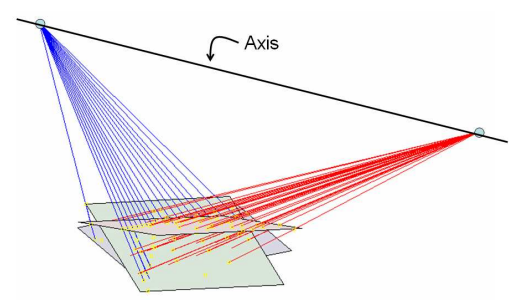

(a)

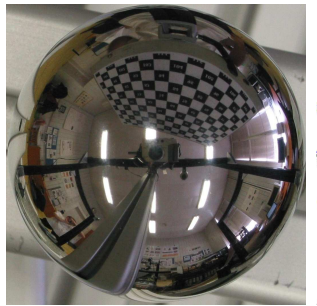

(b)

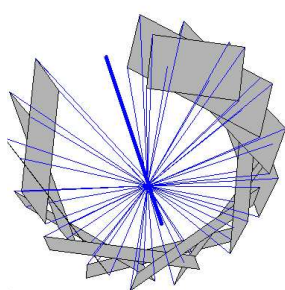

(c)

Fig. 3. Axial calibration: (a) Calibration of a stereo system (b) Image captured by a catadioptric system with a spherical mirror and a perspective camera. (b) Estimated poses of several grids along with the camera axis.

Acknowledgments: We thank Tomášs Pajdla, Branislav Mičuš́́k and Diana Mateus for the data.

\section{References}

1. S. Baker and S. Nayar. A theory of catadioptric image formation. ICCV, 1998.

2. D. Aliaga. Accurate Catadioptric Calibration for Real-size Pose Estimation of Room-size Environments, ICCV, 2001.

3. H. Bakstein and T. Pajdla. An overview of non-central cameras. Computer Vision Winter Workshop, Ljubljana, Slovenia, 2001.

4. Doron Feldman, Tomas Pajdla and Daphna Weinshall. On the Epipolar Geometry of the Crossed-Slits Projection. ICCV, 2003.

5. M.D. Grossberg and S.K. Nayar. A general imaging model and a method for finding its parameters. ICCV, 2001.

6. J. Neumann, C. Fermüller, and Y. Aloimonos. Polydioptric Camera Design and 3D Motion Estimation. CVPR, 2003.

7. T. Pajdla. Stereo with oblique cameras. IJCV, 2002.

8. S. Peleg, M. Ben-Ezra, and Y. Pritch. Omnistereo: Panoramic Stereo Imaging. PAMI, 2001.

9. R. Pless. Using Many Cameras as One. In CVPR, 2003.

10. S. Ramalingam, P. Sturm and S.K. Lodha. Towards Complete Generic Camera Calibration. CVPR, 2005.

11. S. Ramalingam, P. Sturm and S.K. Lodha. Generic calibration of axial cameras. INRIA Research Report, France, December 2005.

12. P. Sturm and S. Ramalingam. A generic concept for camera calibration. $E C C V$, 2004.

13. R. Swaminathan, M.D. Grossberg, and S.K. Nayar. A perspective on distortions. CVPR, 2003.

14. B. Micusik and T. Pajdla. Autocalibration and 3D Reconstruction with Noncentral Catadioptric Cameras. CVPR, 2004.

15. R. Gupta and R.I. Hartley. Linear Pushbroom Cameras. PAMI 1997.

16. S. Seitz and J. Kim. The Space of All Stereo Images. IJCV, 2002. 